\title{
Nonlinear softening and hardening nonlocal bending stiffness of an initially curved monolayer graphene
}

\author{
E. Jomehzadeh ${ }^{\mathrm{a}, *}$, M.K. Afshar ${ }^{\mathrm{b}}$, C. Galiotis ${ }^{\mathrm{c}}$, X. Shi ${ }^{\mathrm{d}}$, N.M. Pugno ${ }^{\mathrm{e}, \mathrm{f}, *}$ \\ a Department of Mechanical Engineering, Graduate University of Advanced Technology, 7631133131 Kerman, Iran \\ b Department of Orthodontic, School of Dentistry, Kerman University of Medical Sciences, 7618759689 Kerman, Iran \\ ${ }^{\text {c }}$ FORTH/ICEHT and Department of Material Science, University of Patras, 26504 Patras, Greece \\ d State Key Laboratory of Nonlinear Mechanics, Institute of Mechanics, Chinese Academy of Sciences, Beijing 100190, China \\ e Laboratory of Bio-Inspired E' Graphene Nanomechanics, Department of Civil, Environmental and Mechanical Engineering, Università di Trento, via Mesiano, \\ 77, I-38123 Trento, Italy \\ ${ }^{\mathrm{f}}$ Center for Materials and Microsystems, Fondazione Bruno Kessler, Via Sommarive 18, I-38123 Povo (Trento), Italy
}

\section{A R T I C L E I N F O}

Available online 20 May 2013

Keywords:

Graphene sheet

Softening

Hardening

Nonlinear behavior

Small length scale

\begin{abstract}
A B S T R A C T
In the present article, the governing nonlinear nonlocal elastic equations are obtained for a monolayer graphene with an initial curvature and the related softening and hardening bending stiffness is analytically calculated. The effects of large deformation, initial curvature, discreteness and direction of chiral vector on the bending stiffness of the monolayer graphene are discussed in detail. A behavior more complex than previously reported in the literature emerges. It is found that the bending stiffness of graphene strongly depends on the initial configuration, showing not obvious maxima and minima, and suggesting the possibility of a smart tuning.
\end{abstract}

(c) 2013 Elsevier Ltd. All rights reserved.

\section{Introduction}

Graphene, the thinnest two-dimensional material, has attracted remarkable considerations since it was first isolated. Its unique electrical, mechanical and thermal properties enable graphene to be used for the development of superconductive devices for the micro-electro-mechanical system (MEMS), the nanoelectro-mechanical system (NEMS) and bioengineering applications [1]. High stiffness, strength and enhanced electrical conductivity of the graphene sheets may result in composites with tailored physical and mechanical properties $[2,3]$.

Determining the elastic properties and response behaviors of graphene sheets have been one of the most hotly disputed areas in recent years. A great deal of research has been conducted to explore the promising properties of graphene sheets by different approaches. The analysis methods of the nanostructures may be classified into four main categories; experiments [4,5], first principles of quantum mechanics [6,7], molecular simulations (MM) $[8,9]$ and continuum modeling [10,11].

Since experiments and quantum mechanical simulations are more difficult and expensive, it could be more convenient to model graphene with other methods. Huang et al. [12] established an

\footnotetext{
* Corresponding authors. Tel.: +98 3426226613; fax: +98 3426226617.

E-mail addresses: e.jomehzadeh@kgut.ac.ir (E. Jomehzadeh), nicola. pugno@unitn.it (N.M. Pugno).
}

analytical approach to bypass atomistic simulations and determined the tension and bending rigidities of graphene and carbon nanotube directly from the interatomic potential. They obtained the thickness and elastic properties and also showed that the bending rigidity is not constant and depends on the type of loading. Pugno [13] calculated stiffness reduction of graphene and nanotubes due to the presence of defects, with specified size, shape, and number. Lu et al. [14] calculated the elastic bending modulus of a graphene layer based on an empirical potential for solid-state carbon atoms. They noted an intrinsic coupling between bending and in-plane strain for graphene sheets rolled into carbon nanotubes and shown a slight nonlinearity and anisotropy in material properties of these structures. Jiang et al. [15] developed a Tersoff-Brenner potential for modeling a graphene sheet by non-linear interactions. They concluded that a large piece of graphene can be regarded as a thin plate by considering the nonlinear effects in the strain components. Scarpa et al. [16] studied the out of plane bending behavior of single layer graphene sheets subjected to point loading by using an atomistic-continuum model.

It should be noted that the atomistic methods are limited to systems with a small number of molecules and atoms and are therefore restricted to small-scale modeling. Hence, development of appropriate continuum models for nanostructures is an important issue. Arroyo and Belytschko [17,18] presented two pioneering works for directly extending the Born rule to the case of crystalline films one atom thick deforming in higher dimensional spaces. They constructed continuum models for one atom thick crystalline film. The Born rule was extended to a film deformation and they showed 
that with an exponential map for finite deformation, the behavior of a continuum membrane mimics the MM model. Cadelano et al. [19] proposed a nonlinear form for constitutive stress-strain relations and calculated the nonlinear elastic moduli for both small and large deformations. Lu and Huang [20] presented a theoretical framework of nonlinear continuum mechanics for two-dimensional graphene sheets under both in-plane and bending deformation. They obtained the cylindrical bending for a initially flat graphene sheet by setting the tube radius equal to length $/ 2 \pi$ in order to uncouple the bending and stretching.

Recent studies have shown significant size-effects in the mechanical properties of nano-structures [21,22]. The classical continuum theories, on the other hand, lack the capability of representing such effects since they do not include any internal length scale. Consequently, these theories are expected to fail when the specimen size becomes comparable with the internal length scale(s) of the material. Therefore, continuum models need to be extended to consider the scale effect in nanomaterial studies. This can be accomplished through proposing nonlocal continuum mechanics models $[23,24]$. Nonlocal elasticity theory accounts the small scale effect by specifying the stress at a reference point as a functional of the strain field at every point in the body. Chen et al. [25] showed that the modeling of nano-structures based on the nonlocal continuum theory is physically reasonable from the atomistic viewpoint of lattice dynamics and molecular dynamics simulations. Recently, lots of studies have been carried out on the analysis of nanostructures by using the nonlocal elasticity theory [26-28].

It was shown in literature that the bending rigidity of a nanostructure is not a constant and depends on the type of loading $[12,29]$. Also, bending stiffness plays an important role for defining the structural stability and mechanical parameters of structures. Moreover, when the graphene sheet rolls into a cylindrical carbon nanotube, the effects of curvature and orientation of chiral vector on the bending stiffness become significant and should be studied. Here, the bending stiffness of a monolayer graphene sheet is calculated using an analytical approach. Since in most cases the stress free configuration of the graphene sheets is not in the completely flat mode, the monolayer graphene is assumed to have an initial curvature. The nonlinear strain-displacement relations are considered to account large deformations. Also, the anisotropic material properties are considered for monolayer graphene. The nonlinear sized-dependent governing equilibrium equations are obtained using the minimum of total potential energy and the bending stiffness of the monolayer graphene under one directional pure bending is analytically calculated. Finally, the effects of initial curvature, chiral angle and cylindrical curvature on the bending stiffness of the graphene layer are thus quantified.

\section{Sized-dependent equations of a monolayer graphene}

Consider a single layered graphene sheet under pure bending moments in the $x$-direction (Fig. 1). The graphene sheet is modeled as a thin orthotropic continuum plate with a thickness $h$ and the coordinate system is located at the middle of the plate in the reference state. The displacement components of an arbitrary point of the sheet can be expressed as

$\tilde{u}=u(x, y)-z \frac{\partial w(x, y)}{\partial x}$

$\tilde{v}=v(x, y)-z \frac{\partial w(x, y)}{\partial y}$

$\tilde{w}=w(x, y)+\bar{w}(x, y)$

where $\bar{w}$ is the initial displacement of the midplane in transverse direction. The initial displacement may occur due to an existing imperfection in the graphene layer or because of energy

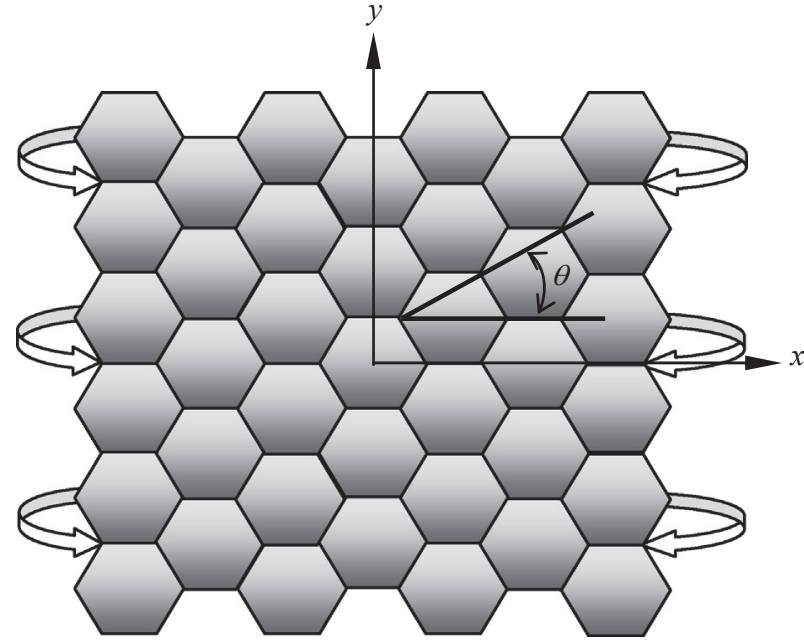

Fig. 1. A schematic geometry of a monolayer graphene under pure bending.

minimization of the graphene. Also, there is an initial configuration in folded and crumpled graphene sheets. In these cases, the graphene layer has no complete initial flat shape. Since the graphene is more flexible in the transverse direction, it is more likely to experience large deformation under a specific load. Also, because of its high in-plane stiffness, the in-plane stretching forces play an important role and cannot be neglected. The nonlinear relations of strain-displacement can be described as

$\varepsilon_{i j}=\frac{1}{2}\left(\frac{\partial u_{i}}{\partial x_{j}}+\frac{\partial u_{j}}{\partial x_{i}}\right)+\frac{1}{2}\left(\frac{\partial u_{m}}{\partial x_{j}} \frac{\partial u_{m}}{\partial x_{j}}\right)$

For moderately large deformations, linearized expressions for the curvature are still valid while the in-plane forces redistribute due to deflections. With the Foppl-Von Karman approximation, the geometrically nonlinear effects arise only from the large rotations that accompany large deflections. Based on this assumption, the straindisplacement relations for displacement (1) can be expressed as [30]

$\varepsilon_{x}=\frac{\partial \tilde{u}}{\partial x}+\frac{1}{2}\left(\frac{\partial \tilde{w}}{\partial x}\right)^{2}$
$\varepsilon_{y}=\frac{\partial \tilde{v}}{\partial y}+\frac{1}{2}\left(\frac{\partial \tilde{w}}{\partial y}\right)$
$\gamma_{x y}=\frac{\partial \tilde{u}}{\partial y}+\frac{\partial \tilde{v}}{\partial x}+\frac{\partial \tilde{w}}{\partial x} \frac{\partial \tilde{w}}{\partial y}$

with this expression, the square derivatives of in-plane displacements neglect with respect to square derivatives of transverse displacement. Also, it is assumed that the graphene sheet is stress free at the undeformed configuration and the initial displacement does not cause the strain at the initial state, i.e. the square derivative of initial curvature is neglected. By considering this assumption and using Eqs. (1) and (3), the strain-displacement relations can be obtained as

$\varepsilon_{x}=\varepsilon_{x 0}+z \kappa_{x}$
$\varepsilon_{y}=\varepsilon_{y 0}+z \kappa_{y}$
$\gamma_{x y}=\gamma_{x y 0}+z \kappa_{x y}$

where in-plane strains and curvature parameters are defined as

$\left\{\begin{array}{l}\varepsilon_{x 0} \\ \varepsilon_{y 0} \\ \gamma_{x y 0}\end{array}\right\}=\left\{\begin{array}{l}\frac{\partial u}{\partial x}+\frac{1}{2}\left(\frac{\partial w}{\partial x}\right)^{2}+\frac{\partial w}{\partial x} \frac{\partial \bar{w}}{\partial x} \\ \frac{\partial v}{\partial y}+\frac{1}{2}\left(\frac{\partial w}{\partial y}\right)^{2}+\frac{\partial w}{\partial y} \frac{\partial \bar{w}}{\partial y} \\ \frac{\partial u}{\partial y}+\frac{\partial v}{\partial x}+\frac{\partial w}{\partial x} \frac{\partial w}{\partial y}+\frac{\partial w}{\partial x} \frac{\partial \bar{w}}{\partial y}+\frac{\partial \bar{w}}{\partial x} \frac{\partial w}{\partial y}\end{array}\right\}, \quad\left\{\begin{array}{l}\kappa_{x} \\ \kappa_{y} \\ \kappa_{x y}\end{array}\right\}=\left\{\begin{array}{l}-\frac{\partial^{2} w}{\partial x^{2}} \\ -\frac{\partial^{2} w}{\partial y^{2}} \\ -2 \frac{\partial^{2} w}{\partial x \partial y}\end{array}\right\}$ 
It is also clear that the magnitude of higher order terms with respect to $z \kappa_{x}, z \kappa_{y}$ and $z \kappa_{x y}$ are considered negligible in the treatment. At the initial configuration, the displacement components $(u, v, w)$ and their derivatives are zero, thus the strain components vanish and therefore the graphene layer is stress free at reference state with an initial deflection.

Expressing the strain and potential energies of the graphene and using the theory of minimum total potential energy, the nonlinear classical equilibrium equations can be obtained. Since the dimensions of the monolayer graphene is in the range of nanometer, it is not reasonable to use the classical equilibrium equations. As the length of a structure is reduced in the range of nanometer, the influence of the long range interatomic and intermolecular forces become significant and cannot be neglected. The classical continuum mechanics cannot capture this effect and therefore would not produce accurate results for size-effects.

In order to consider the small scale effect of interatomic interactions, the nonlocal elasticity theory can be used. According to nonlocal elasticity, the stress in a definite point in a body depends not only on the strain in that point but also on those in the others. Nonlocal continuum mechanics allows one to account for the small length scale effect that becomes significant when dealing with nanostructures. Eringen [23] showed that the nonlocal stress tensor $\left(\sigma^{n l}\right)$ can be related to local stress tensor $(\sigma)$ by the following differential form:

$\sigma^{n l}-\tau^{2} l_{e}^{2} \nabla^{2} \sigma^{n l}=\sigma, \quad \tau=e_{0} l_{i} / l_{e}$

where $e_{0} l_{i}$ is a small scale parameter, $e_{0}$ is a constant appropriate to each material for adjusting the model to match the reliable experimental results, $l_{i}$ is an internal material characteristic length such as $\mathrm{C}-\mathrm{C}$ bond length, $l_{e}$ is the external structural characteristic length and $\nabla^{2}$ is the Laplacian operator [31]. The important advantage of the Eringen approach is that its constitutive relations are differential equations instead of integral equations. In this approach, Fourier transforms of the constitutive moduli are expanded into power series of the wave number and then the integral operators are replaced with differential ones for slow varying field [24]. Also, in this model additional boundary conditions do not appear.

The based equations of nonlocal elasticity have been obtained based on the simple lattice model for isothermal elastic solids consist of discrete atomic mass points attached to each other by springs [24]. Therefore, the nonlocal elasticity theory was derived from the discrete nature of lattice dynamic and can capture the small sized effects. Hence, although the classical plates theory is not appropriate for analysis of nano-structures, the results of the modified plate model by the nonlocal elasticity theory can become closer to accurate results.

Considering the small scale effect and using the theory of minimum potential energy, the nonlocal equilibrium equations can be obtained as

$\frac{\partial N_{x}^{n l}}{\partial x}+\frac{\partial N_{x y}^{n l}}{\partial y}=0$

$\frac{\partial N_{x y}^{n l}}{\partial x}+\frac{\partial N_{y}^{n l}}{\partial y}=0$

$$
\begin{aligned}
\frac{\partial^{2} M_{x}^{n l}}{\partial x^{2}} & +2 \frac{\partial^{2} M_{x y}^{n l}}{\partial x \partial y}+\frac{\partial^{2} M_{y}^{n l}}{\partial y^{2}}+\frac{\partial}{\partial x}\left[N_{x}^{n l}\left(\frac{\partial w}{\partial x}+\frac{\partial \bar{w}}{\partial x}\right)+N_{x y}^{n l}\left(\frac{\partial w}{\partial y}+\frac{\partial \bar{w}}{\partial y}\right)\right] \\
& +\frac{\partial}{\partial y}\left[N_{x y}^{n l}\left(\frac{\partial w}{\partial x}+\frac{\partial \bar{w}}{\partial x}\right)+N_{y}^{n l}\left(\frac{\partial w}{\partial y}+\frac{\partial \bar{w}}{\partial y}\right)\right]+p(x, y)=0
\end{aligned}
$$

where $p(x, y)$ is the external pressure and is zero in our problem. By multiplying both sides of the above equations by $\left(1-\left(e_{0} l_{i}\right)^{2} \nabla^{2}\right)$ and considering relation (6), yields

$$
\begin{aligned}
& \frac{\partial N_{x}}{\partial x}+ \frac{\partial N_{x y}}{\partial y}=0 \\
& \frac{\partial N_{x y}}{\partial x}+\frac{\partial N_{y}}{\partial y}=0 \\
& \frac{\partial^{2} M_{x}}{\partial x^{2}}+2 \frac{\partial^{2} M_{x y}}{\partial x \partial y}+\frac{\partial^{2} M_{y}}{\partial y^{2}}+\left(1-\left(e_{0} l_{i}\right)^{2} \nabla^{2}\right) \frac{\partial}{\partial x} \\
& {\left[N_{x}^{n l}\left(\frac{\partial w}{\partial x}+\frac{\partial \bar{w}}{\partial x}\right)+N_{x y}^{n l}\left(\frac{\partial w}{\partial y}+\frac{\partial \bar{w}}{\partial y}\right)\right]+\left(1-\left(e_{0} l_{i}\right)^{2} \nabla^{2}\right) \frac{\partial}{\partial y} } \\
& {\left[N_{x y}^{n l}\left(\frac{\partial w}{\partial x}+\frac{\partial \bar{w}}{\partial x}\right)+N_{y}^{n l}\left(\frac{\partial w}{\partial y}+\frac{\partial \bar{w}}{\partial y}\right)\right]+\left(1-\left(e_{0} l_{i}\right)^{2} \nabla^{2}\right) p(x, y)=0 }
\end{aligned}
$$

where $N_{x}, N_{y}, N_{x y}$ are the in-plane resultant forces and $M_{x}, M_{y}, M_{x y}$ are the resultant moments of monolayer graphene.

Due to the hexagonal structure of the unit cells in graphene sheets, it can be considered as an orthotropic material especially for large deformations. For a two-dimensional orthotropic material, the local stress tensor can be related to the strain tensor as

$\left\{\begin{array}{l}\sigma_{x} \\ \sigma_{y} \\ \sigma_{x y}\end{array}\right\}=\left[\begin{array}{lll}Q_{11} & Q_{12} & Q_{16} \\ Q_{12} & Q_{22} & Q_{26} \\ Q_{16} & Q_{26} & Q_{66}\end{array}\right]\left\{\begin{array}{l}\varepsilon_{x} \\ \varepsilon_{y} \\ \gamma_{x y}\end{array}\right\}$

Components $Q_{i j}(i, j=1,2,6)$ are the elastic properties of the graphene sheet. These components depend on the direction of the chiral vector. By indicating with $\theta$ the angle between zigzag and $x$-direction (Fig. 1), the elastic components can be defined as

$$
\left[\begin{array}{l}
Q_{11} \\
Q_{22} \\
Q_{12} \\
Q_{66} \\
Q_{16} \\
Q_{26}
\end{array}\right]=\left[\begin{array}{cccc}
c^{4} & s^{4} & 2 c^{2} s^{2} & 4 c^{2} s^{2} \\
s^{4} & c^{4} & 2 c^{2} s^{2} & 4 c^{2} s^{2} \\
c^{2} s^{2} & c^{2} s^{2} & c^{4}+s^{4} & -4 c^{2} s^{2} \\
c^{2} s^{2} & c^{2} s^{2} & -2 c^{2} s^{2} & \left(c^{2}-s^{2}\right)^{2} \\
c^{3} s & -c s^{3} & c s^{3}-c^{3} s & 2 c s\left(s^{2}-c^{2}\right) \\
c s^{3} & -c^{3} s & c^{3} s-c s^{3} & 2 c s\left(c^{2}-s^{2}\right)
\end{array}\right]\left[\begin{array}{l}
E_{11} /\left(1-\nu_{12} \nu_{21}\right) \\
E_{22} /\left(1-\nu_{12} \nu_{21}\right) \\
\nu_{21} E_{11} /\left(1-\nu_{12} \nu_{21}\right) \\
E_{11} /\left(2+2 \nu_{12}\right)
\end{array}\right]
$$

where $E_{11}$ and $E_{22}$ are respectively the Young modulus in zigzag and armchair directions and $\nu$ is the Poisson ratio. Also, $s$ and $c$ denote $\sin \theta$ and $\cos \theta$ respectively (in which $\theta=0$ represents a zigzag graphene sheet and $\theta=\pi / 2$ corresponds to an armchair graphene layer).

Expressing the resultant forces and moments in terms of strain components, yields

$\left\{\begin{array}{l}N \\ M\end{array}\right\}=\left[\begin{array}{ll}{[A]} & {[B]} \\ {[B]} & {[D]}\end{array}\right]\left\{\begin{array}{l}\varepsilon_{0} \\ \kappa\end{array}\right\}$

where $[A]$ and $[D]$ are called the matrices of stretching and bending stiffness, respectively and $[B]$ is the matrix of stretching-bending coupling stiffness, namely

$\left(A_{i j}, B_{i j}, D_{i j}\right)=\int_{-h / 2}^{h / 2} Q_{i j}\left(1, z, z^{2}\right) d z$

Obtaining the force and moments resultants for an anisotropic graphene sheet and introducing the stress function $\varphi$ as

$N_{x}=\frac{\partial^{2} \varphi}{\partial y^{2}}, \quad N_{y}=\frac{\partial^{2} \varphi}{\partial x^{2}}, \quad N_{x y}=-\frac{\partial^{2} \varphi}{\partial x \partial y}$

and considering the small scale effect, the equilibrium equations (8) are converted into the following equation:

$$
\begin{aligned}
D_{11}^{*} \frac{\partial^{4} w}{\partial x^{4}} & +2\left(D_{12}^{*}+2 D_{66}^{*}\right) \frac{\partial^{4} w}{\partial x^{2} \partial y^{2}}+D_{22}^{*} \frac{\partial^{4} w}{\partial y^{4}}+4 D_{16}^{*} \frac{\partial^{4} w}{\partial x^{3} \partial y} \\
& +4 D_{26}^{*} \frac{\partial^{4} w}{\partial x \partial y^{3}}+B_{21}^{*} \frac{\partial^{4} \varphi}{\partial x^{4}}+B_{12}^{*} \frac{\partial^{4} \varphi}{\partial y^{4}}+\left(B_{11}^{*}+B_{22}^{*}-2 B_{66}^{*}\right) \frac{\partial^{4} \varphi}{\partial x^{2} \partial y^{2}}
\end{aligned}
$$




$$
\begin{aligned}
& +\left(2 B_{26}^{*}-B_{61}^{*}\right) \frac{\partial^{4} \varphi}{\partial x^{3} \partial y}+\left(2 B_{16}^{*}-B_{62}^{*}\right) \frac{\partial^{4} \varphi}{\partial x \partial y^{3}}-\left(1-\left(e_{0} l_{i}\right)^{2} \nabla^{2}\right) \\
& {\left[\frac{\partial^{2} \varphi}{\partial y^{2}} \frac{\partial^{2}}{\partial x^{2}}(w+\bar{w})-2 \frac{\partial^{2} \varphi}{\partial x \partial y} \frac{\partial^{2}}{\partial x \partial y}(w+\bar{w})\right.} \\
& \left.+\frac{\partial^{2} \varphi}{\partial x^{2}} \frac{\partial^{2}}{\partial y^{2}}(w+\bar{w})\right]=0
\end{aligned}
$$

where $D_{i j}^{*}=D_{i j}-B_{i j} A_{i j}^{*} B_{i j}$ in which $A^{*}$ is the inverse of matrix $A$. The compatibility equation of the strain components of the middle surface is written as [30]

$$
\begin{gathered}
\varepsilon_{x 0, y y}+\varepsilon_{y 0, x x}-2 \varepsilon_{x y 0, x y}=\left(\frac{\partial^{2} w}{\partial x \partial y}\right)^{2}-\frac{\partial^{2} w}{\partial x^{2}} \frac{\partial^{2} w}{\partial y^{2}} \\
+2 \frac{\partial^{2} w}{\partial x \partial y} \frac{\partial^{2} \bar{w}}{\partial x \partial y}-\frac{\partial^{2} w}{\partial y^{2}} \frac{\partial^{2} \bar{w}}{\partial x^{2}}-\frac{\partial^{2} w}{\partial x^{2}} \frac{\partial^{2} \bar{w}}{\partial y^{2}}
\end{gathered}
$$

Using Eq. (11), the strains components can be expressed in terms of the stress function as

$\left\{\begin{array}{l}\varepsilon_{0} \\ M\end{array}\right\}=\left[\begin{array}{ll}{[A]^{-1}} & -[A]^{-1}[B] \\ {[B][A]^{-1}} & {[D]-[B][A]^{-1}[B]}\end{array}\right]\left\{\begin{array}{l}N \\ \kappa\end{array}\right\}=\left[\begin{array}{ll}A^{*} & B^{*} \\ -B^{* T} & D^{*}\end{array}\right]\left\{\begin{array}{l}N \\ \kappa\end{array}\right\}$

Substituting Eq. (16) into compatibility Eq. (15) and using Eq. (13), yields

$$
\begin{aligned}
A_{22}^{*} \frac{\partial^{4} \varphi}{\partial x^{4}} & +\left(A_{66}^{*}+2 A_{12}^{*}\right) \frac{\partial^{4} \varphi}{\partial x^{2} \partial y^{2}}+A_{11}^{*} \frac{\partial^{4} \varphi}{\partial y^{4}} \\
& -2 A_{16}^{*} \frac{\partial^{4} \varphi}{\partial x \partial y^{3}}-2 A_{26}^{*} \frac{\partial^{4} \varphi}{\partial x^{3} \partial y}=\left(\frac{\partial^{2} w}{\partial x \partial y}\right)^{2}-\frac{\partial^{2} w}{\partial x^{2}} \frac{\partial^{2} w}{\partial y^{2}} \\
& +2 \frac{\partial^{2} w}{\partial x \partial y} \frac{\partial^{2} \bar{w}}{\partial x \partial y}-\frac{\partial^{2} w}{\partial y^{2}} \frac{\partial^{2} \bar{w}}{\partial x^{2}}-\frac{\partial^{2} w}{\partial x^{2}} \frac{\partial^{2} \bar{w}}{\partial y^{2}}+B_{21}^{*} \frac{\partial^{4} w}{\partial x^{4}}+B_{12}^{*} \frac{\partial^{4} w}{\partial y^{4}} \\
& +\left(B_{11}^{*}+B_{22}^{*}-2 B_{66}^{*}\right) \frac{\partial^{4} w}{\partial x^{2} \partial y^{2}}+\left(2 B_{26}^{*}-B_{61}^{*}\right) \frac{\partial^{4} w}{\partial x^{3} \partial y} \\
& +\left(2 B_{16}^{*}-B_{62}^{*}\right) \frac{\partial^{4} w}{\partial x \partial y^{3}}
\end{aligned}
$$

Eqs. (14) and (17) are the starting equations for studying the nonlinear behavior of graphene sheets. Also, the in-plane displacement components can be expressed in terms of the transverse deflection and stress function by the help of Eqs. (5), (13) and (16) as

$u=\int_{0}^{x}\left(A_{11}^{*} \frac{\partial^{2} \varphi}{\partial x^{2}}+A_{12}^{*} \frac{\partial^{2} \varphi}{\partial y^{2}}+A_{16}^{*} \frac{\partial^{2} \varphi}{\partial x \partial y}-\frac{1}{2}\left(\frac{\partial w}{\partial x}\right)^{2}-\frac{\partial w}{\partial x} \frac{\partial \bar{w}}{\partial x}\right) d x$

$v=\int_{0}^{y}\left(A_{12}^{*} \frac{\partial^{2} \varphi}{\partial x^{2}}+A_{22}^{*} \frac{\partial^{2} \varphi}{\partial y^{2}}+A_{26}^{*} \frac{\partial^{2} \varphi}{\partial x \partial y}-\frac{1}{2}\left(\frac{\partial w}{\partial y}\right)^{2}-\frac{\partial w}{\partial y} \frac{\partial \bar{w}}{\partial y}\right) d y$

Thus, by determining the transverse displacement and stress function, the in-plane displacements can also be defined.

\section{Bending stiffness of a monolayer graphene}

Let us consider a monolayer graphene that is subjected to bending moments in the $x$-direction (longitudinal direction) and have free edges in the $y$-direction (lateral direction). For large values of moments, this problem implies a nonlinear large deformations and it does not have simple solution. Here we would like to obtain the value of the required moment to create a specific type of deformation. Since every cross section of the graphene will deform identically in this type of problem, it is reasonable to focus on the central portion of the graphene layer that is away from the applied moments. In this region, the variation of the transverse deflection with respect to the lateral coordinate $y$ is independent of the longitudinal coordinate $x$. Moreover, at the central portion of the graphene sheet, the applied moments can be assumed to cause a constant curvature in the longitudinal direction. Accordingly, the transverse deflection is assumed to be in the following form:

$w(x, y)=w_{1}(y)+\frac{x^{2}}{2 R_{x}}$

where $R_{x}$ is the known radius of curvature in the longitudinal direction $\left(\kappa_{x}=1 / R_{x}\right)$. Also, $w_{1}(y)$ shows the variation of the transverse displacement on the lateral direction.

The initial deviation of the graphene from its flat configuration can be considered as a mild half-wave type. Here, the initial imperfection in the graphene is taken to be in the form of

$\bar{w}(x, y)=\frac{x^{2}}{2 \bar{R}_{x}}+\frac{y^{2}}{2 \bar{R}_{y}}$

where the parameters $\bar{R}_{x}$ and $\bar{R}_{y}$ represent the longitudinal and lateral radii of initial curvature. Substituting the above relations into Eqs. (14) and (17), yields

$$
\begin{aligned}
D_{22}^{*} \frac{\partial^{4} w_{1}}{\partial y^{4}} & -\left(\frac{1}{R_{x}}+\frac{1}{\bar{R}_{x}}\right) \frac{\partial^{2} \varphi}{\partial y^{2}}-\left(\frac{1}{\bar{R}_{y}}+\frac{\partial^{2} w_{1}}{\partial y^{2}}\right) \frac{\partial^{2} \varphi}{\partial x^{2}} \\
& +\left(e_{0} l_{i}\right)^{2}\left[\left(\frac{1}{R_{x}}+\frac{1}{\bar{R}_{x}}\right) \frac{\partial^{4} \varphi}{\partial x^{2} \partial y^{2}}+2 \frac{\partial^{3} w_{1}}{\partial y^{3}} \frac{\partial^{3} \varphi}{\partial x^{2} \partial y}\right. \\
& +\left(\frac{1}{R_{y 0}}+\frac{\partial^{2} w_{1}}{\partial y^{2}}\right) \frac{\partial^{4} \varphi}{\partial x^{4}}+\left(\frac{1}{R_{x}}+\frac{1}{\bar{R}_{x}}\right) \frac{\partial^{4} \varphi}{\partial y^{4}}+\frac{\partial^{4} w_{1}}{\partial y^{4}} \frac{\partial^{2} \varphi}{\partial x^{2}} \\
& \left.+\left(\frac{1}{\bar{R}_{y}}+\frac{\partial^{2} w_{1}}{\partial y^{2}}\right) \frac{\partial^{4} \varphi}{\partial x^{2} \partial y^{2}}\right]=0 \\
A_{22}^{*} \frac{\partial^{4} \varphi}{\partial x^{4}}+ & \left(A_{66}^{*}+2 A_{12}^{*}\right) \frac{\partial^{4} \varphi}{\partial x^{2} \partial y^{2}}+A_{11}^{*} \frac{\partial^{4} \varphi}{\partial y^{4}}-2 A_{16}^{*} \frac{\partial^{4} \varphi}{\partial x \partial y^{3}} \\
- & 2 A_{26}^{*} \frac{\partial^{4} \varphi}{\partial x^{3} \partial y}+\left(\frac{1}{R_{x}}+\frac{1}{\bar{R}_{x}}\right) \frac{\partial^{2} w}{\partial y^{2}}+\frac{1}{R_{x} \bar{R}_{y}}=0
\end{aligned}
$$

It should be noted that the components of stretching-bending coupling stiffness matrix are zero for a monolayer graphene sheet. As stated before, our attention concerns in the region away from the applied moments and thus the longitudinal curvature is constant and the cross sections deform nearly uniformly in the longitudinal direction. Therefore, it is quite reasonable to assume that the stress variation is independent from the longitudinal coordinate $\left(N_{x}=N_{x}(y)\right.$ ). In addition, it is clear that the magnitude of terms of higher order with respect to $z \kappa_{x}, z \kappa_{y}$ and $z \kappa_{x y}$ are considered negligible in the treatment and the lateral or minor curvature is small compared to the longitudinal or major curvature along the moments direction $\left(\left(\partial^{2} w_{1} / \partial y^{2}\right) / \ll\left(1 / R_{x}\right)\right)$. Employing these simplifications and using Eqs. (13) and (21), it can be obtained

$N_{x}=-\left(\frac{1}{R_{x}}+\frac{1}{R_{x 0}}\right) \frac{1}{A_{11}^{*}} w_{1}(y)-\frac{1}{2 A_{11}^{*} R_{x} R_{y 0}} y^{2}+C_{1} y+C_{2}$

Since the geometry and loading of the graphene are symmetric with respect to coordinate directions, the unknown variable $C_{1}$ is zero. Substituting Eq. (22) into Eq. (21a), yields

$$
\begin{gathered}
D_{22}^{*} \frac{\partial^{4} w_{1}}{\partial y^{4}}-\frac{2 \bar{R}_{x}^{2} \bar{R}_{y}+2 R_{x}^{2} \bar{R}_{y}+4 R_{x} \bar{R}_{x} \bar{R}_{y}}{2 A_{11}^{*} R_{x}^{2} \bar{R}_{x}^{2} \bar{R}_{Y}}\left[\left(e_{0} l_{i}\right)^{2} \frac{\partial^{2} w_{1}}{\partial y^{2}}+w_{1}\right] \\
+\frac{\bar{R}_{x}^{2}+R_{x} \bar{R}}{2 A_{11}^{*} R_{x}^{2} \bar{R}_{x}^{2} \bar{R}_{Y}}\left[\left(e_{0} l_{i}\right)^{2}+y^{2}\right]-\frac{R_{x}+\bar{R}_{x}}{R_{x} \bar{R}_{x}} C_{2}=0
\end{gathered}
$$

Solving the above equation and using Eq. (19), the transverse deflection of a symmetric graphene sheet can be obtained as $w(x, y)=C_{3} \sinh (\operatorname{Re}(\lambda)) \sin (\operatorname{Im}(\lambda))+C_{4} \cosh (\operatorname{Re}(\lambda)) \cos (\operatorname{Im}(\lambda))$ 


$$
+\frac{\bar{R}_{x}\left(2\left(e_{0} l_{i}\right)^{2}+y^{2}\right)}{2 \bar{R}_{y}\left(R_{x}+\bar{R}_{x}\right)}+\frac{x^{2}}{2 R_{x}}
$$

where $\lambda$ is the root of characteristic equation (23) (Re and Im represent the real and imaginary parts, respectively). It should be noted that the summation of Eq. (24) and Eq. (20) defines the total transverse deflection of the monolayer graphene.

In order to obtain the unknown coefficients $C_{2}, C_{3}$ and $C_{4}$, the free boundary conditions at the edge of the graphene in the $y$-direction should be imposed. The boundary conditions can be written as

$N_{y}^{n l}=N_{x y}^{n l}=\frac{\partial M_{y}^{n l}}{\partial y}+2 \frac{\partial M_{x y}^{n l}}{\partial x}=M_{y}^{n l}=0 \quad$ at $y= \pm b / 2$

in which the two first boundary conditions are identically satisfied by an appropriate coefficient $C_{2}$. It should be noticeable that forces and moments in Eq. (25) are the nonlocal resultants, i.e.

$M_{y}^{n l}-\left(e_{0} l_{i}\right)^{2} \nabla^{2} M_{y}^{n l}=M_{y}$

Some studies in the literature neglect the local forces and moments at the boundaries instead of nonlocal ones. In order to find more accurate results, it is reasonable to impose the nonlocal boundary conditions. To this end, it can be shown from Eq. (26) that the nonlocal moment resultant can be written as

$$
\begin{aligned}
M_{y}^{n l}= & M_{y}+\left(e_{0} l_{i}\right)^{2} \nabla^{2} M_{y}+\left(e_{0} l_{i}\right)^{4} \nabla^{4} M_{y}+\left(e_{0} l_{i}\right)^{6} \nabla^{6} M_{y} \\
& +\left(e_{0} l_{i}\right)^{8} \nabla^{8} M_{y}+\cdots=\sum_{n=0}\left(e_{0} l_{i}\right)^{2 n} \nabla^{2 n} M_{y}
\end{aligned}
$$

The resultant forces can also be expressed in this way $N_{y}^{n l}=\Sigma_{n=0}\left(e_{0} l_{i}\right)^{2 n} \nabla^{2 n} N_{y}$. By applying the two last boundary conditions in Eq. (25), the two unknown coefficients $C_{3}$ and $C_{4}$ can be obtained and therefore the deflection of the monolayer graphene can be calculated. Finally, the applied moment in the longitudinal direction can be expressed as

$M_{0}=\frac{1}{b} \int_{-b / 2}^{b / 2} \sum_{n=0}\left(e_{0} l_{i}\right)^{2 n} \nabla^{2 n} M_{y} d y$

It can be seen that the above explicit equation can be used to determine what value of applied moment is necessary to produce a given longitudinal curvature in a monolayer graphene. In order to define the bending stiffness of the monolayer graphene, it is only required to differentiate the applied moment in Eq. (28) with respect to the longitudinal curvature $\left(\partial M_{0} / \partial \kappa_{x}\right)$. Also, it can be shown that the total forces of edges in longitudinal direction can be obtained by integrating $N_{x}$ along the $y$-direction and is found to be zero.

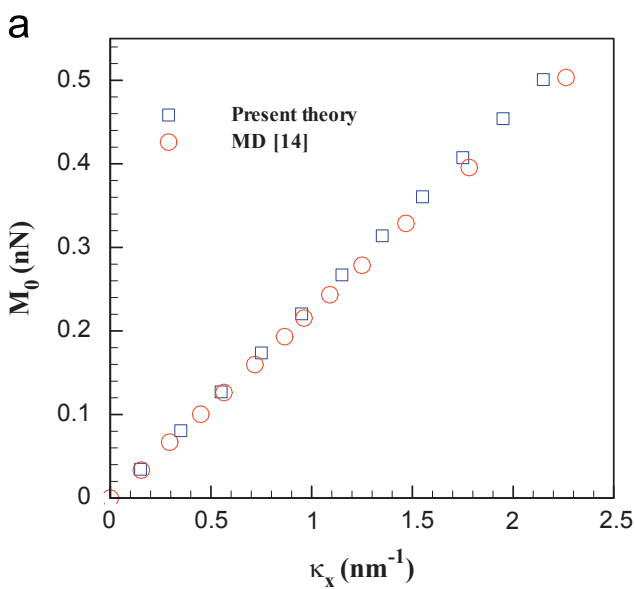

\section{Numerical results}

In this section, the numerical results are presented for a monolayer graphene with an applied curvature in the longitudinal direction. The bending modulus of graphene layer was predicted to be $1.46 \mathrm{eV}$ from ab initio simulation [6] and $1.4 \mathrm{eV}$ from the Brenner potential [14]. In the present study, the following material properties are considered for numerical modeling

$\widehat{D}_{11}=0.234 \mathrm{nN} \mathrm{nm}=1.46 \mathrm{eV} ; \quad \widehat{D}_{22}=0.229 \mathrm{nN} \mathrm{nm}=1.43 \mathrm{eV} ;$

$\nu_{12}=0.149 ; \quad \nu_{21}=0.145$

where $\widehat{D}_{i i}$ is the bending modulus of graphene. The nonlocal parameter is depended on the dimensions of the graphene and it is assumed to be $e_{0} l_{i}=0.1 b$ in numerical results where $b$ is the $\mathrm{C}-\mathrm{C}$ distance.

For verification of the accuracy of the present results, a comparison has been carried out with the results of the second-generation Brenner potential for solid-state carbon atoms of a monolayer graphene [14]. The initial configuration is assumed to be flat. The variation of the bending moment per length is compared with the results of molecular dynamics in Fig. 2 for both zigzag and armchair graphene sheets. It can be seen that the present results are in good agreement with the results of the molecular dynamics simulations using the second-generation of the Brenner potential.

To find the bending stiffness of the monolayer graphene, the slope of the bending moment per unit length with respect to the longitudinal curvature is obtained. The variation of the bending stiffness of the graphene sheet is shown in Fig. 3 for different initial curvatures. It can be seen that the stiffness of graphene does not always have hardening behavior and changes of stiffness are significant for low and medium curvatures. Depending on the initial configuration of the graphene, the bending stiffness may also have a softening behavior. It can be seen that some graphene sheets have the softening bending stiffness in some ranges of curvature. When lateral and longitudinal initial curvatures are not in the same directions, it can be found that the bending stiffness usually decreases by increasing the longitudinal curvature. It can be concluded that the introduction of an initial curvature considerably changes the behavior of the graphene sheet and causes a complex nonlinear behavior for bending stiffness. It is found that the graphene can reach a condition in which further increase of moments increases or decreases the bending stiffness of the graphene with reference to its flat or initial state.

In order to understand the cause of the hardening or softening behavior of bending stiffness, it is appropriate to depict the

b

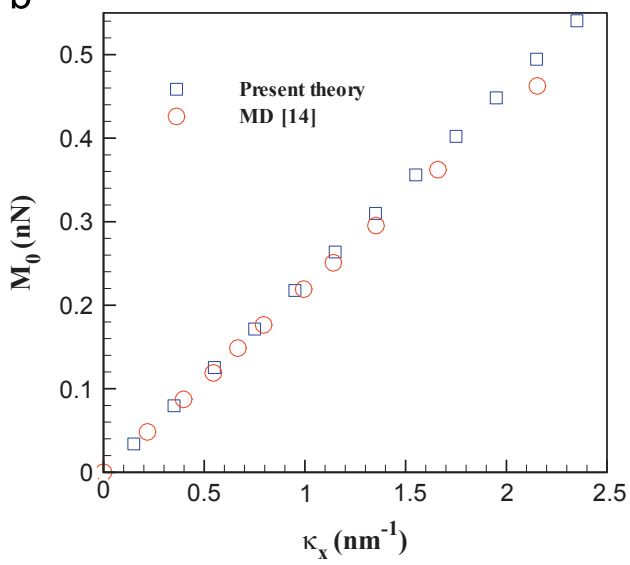

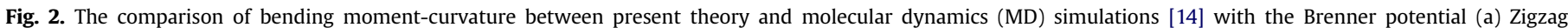
graphene (b) Armchair graphene. 
a

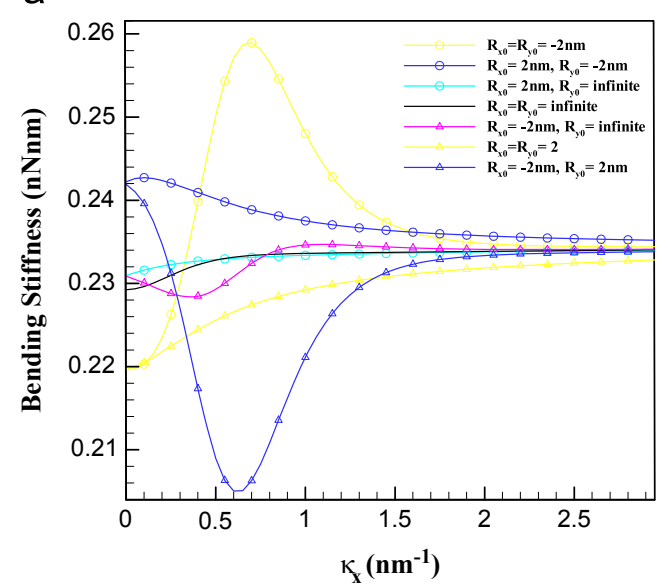

b

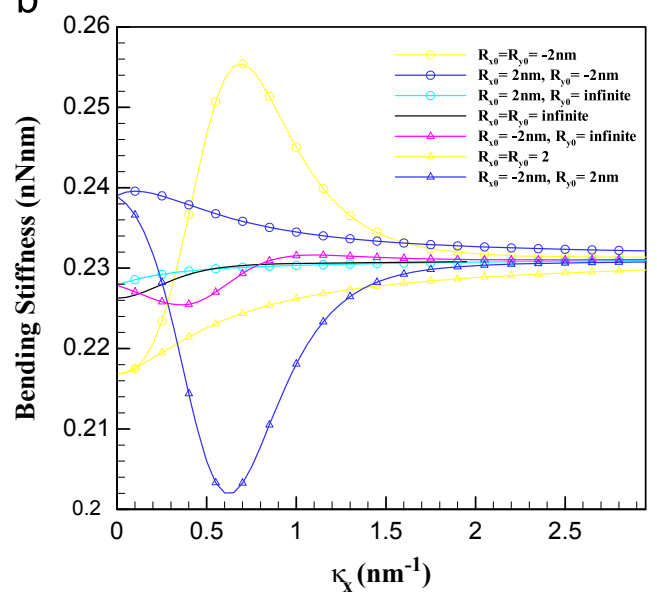

Fig. 3. Variation of bending stiffness of the monolayer graphene versus curvature for (a) Zigzag graphene and (b) Armchair graphene.
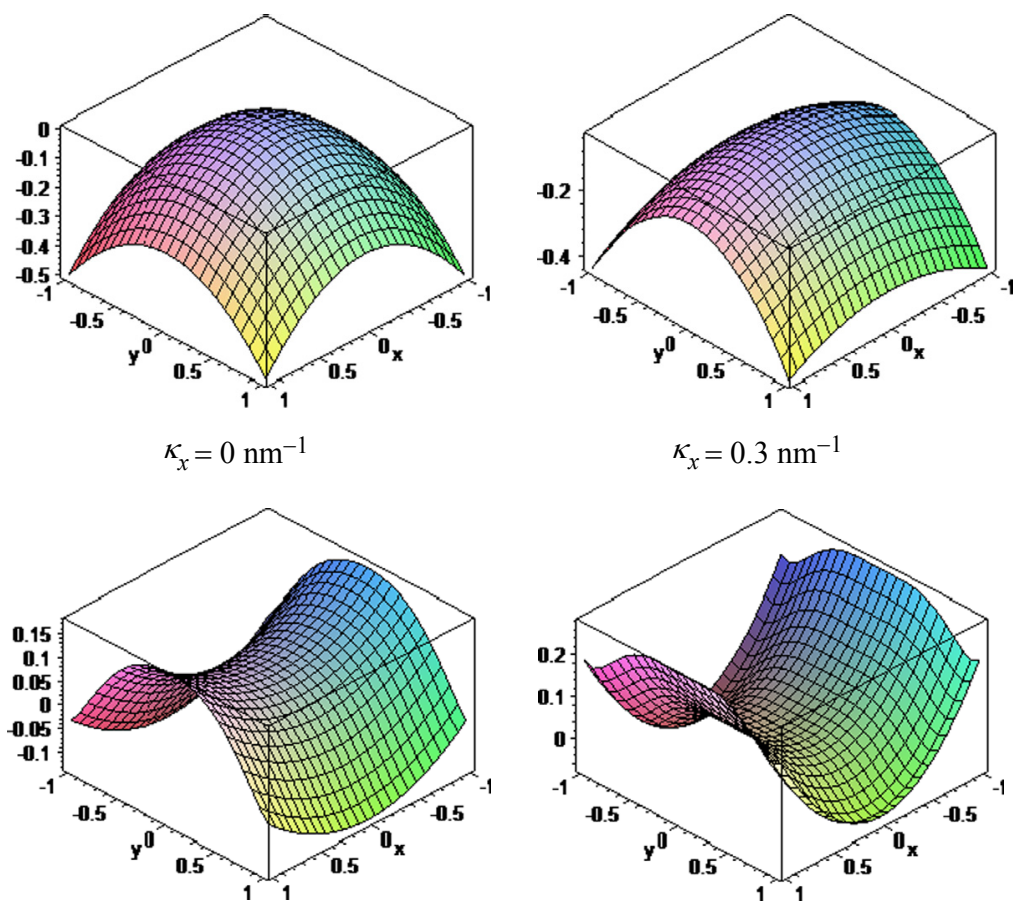

$\kappa_{x}=0.7 \mathrm{~nm}^{-1}$

$\kappa_{x}=1 \mathrm{~nm}^{-1}$
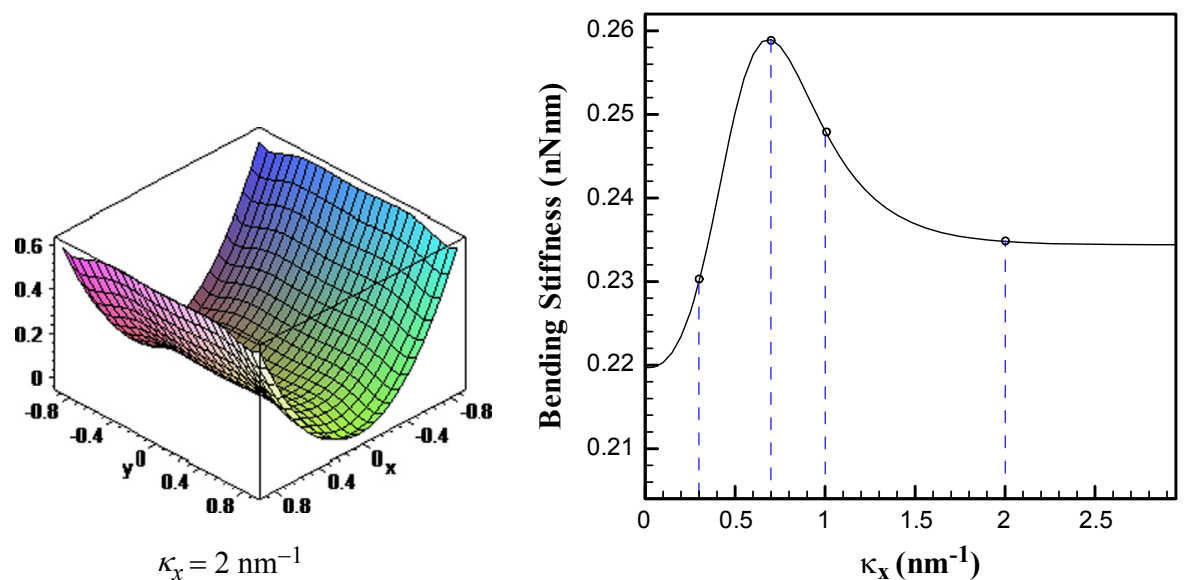

Fig. 4. The deformed shapes of monolayer graphene for different longitudinal curvatures $R_{x 0}=R_{y 0}=-2 \mathrm{~nm}$. 
deformed shapes of a monolayer graphene for different values of the applied moments and curvatures. The deformed shapes are shown for an initially curved zigzag graphene $\left(R_{x 0}=R_{y 0}=-2 \mathrm{~nm}\right)$ for different values of curvature in Fig. 4. It can be found that for this geometry of graphene, the stiffness has hardening behavior for $\kappa_{x}<0.7 \mathrm{~nm}^{-1}$ and after that it decreases for increasing the curvature until $\kappa_{x}=2 \mathrm{~nm}^{-1}$. Also, it has nearly the constant stiffness for high values of curvature. As it can be seen in Fig. 4, the applied moment tends to create an opposite curvature in the longitudinal direction with respect to lateral direction for $\kappa_{x}<0.7 \mathrm{~nm}^{-1}$ and this causes an increasing of the moment of inertia and thus of the stiffness. After that, the stiffness is reduced due to flattening in the lateral direction. As the graphene becomes flat in this direction, the moment of inertia of the cross section about its neutral surface decreases and causes the decreasing of the flexural rigidity. Another interesting result is that as the lateral curvature changes its direction around $\kappa_{x}=0.7 \mathrm{~nm}^{-1}$, an elastic instability may occur and cause decreasing of the stiffness in graphene. In addition, the graphene is converted to a cylindrical shape with nearly small lateral curvature for high longitudinal curvatures. In this stage, the variation of the stiffness of graphene is not significant. The tendency of graphene to become flat in lateral direction is due to geometrical nonlinear effects.

The deformed shapes of the graphene sheet with opposite initial curvatures $\left(R_{x 0}=-2 \mathrm{~nm}, R_{y 0}=2 \mathrm{~nm}\right)$ are also shown in Fig. 5 . It can be seen that this geometry of graphene has a softening behavior for $\kappa_{x}<0.6 \mathrm{~nm}^{-1}$. As it can be seen in Fig. 5, the applied moment makes the graphene flat in the longitudinal direction for low curvatures and because of this the stiffness decreases. Since the graphene tends to reduce the lateral curvature after its particular value $\left(\kappa_{x}=0.6 \mathrm{~nm}^{-1}\right)$, the stiffness increases by increasing the curvature. As in the previous case, the stiffness converges to a nearly asymptotic value when the lateral curvature diminishes.

In order to study the effect of chiral angle, the variation of the stiffness versus the longitudinal curvature is depicted in Fig. 6 for different directions of material. It can be seen that the highest bending stiffness is in the zigzag direction and the effect of chiral angle becomes more significant for higher curvatures.
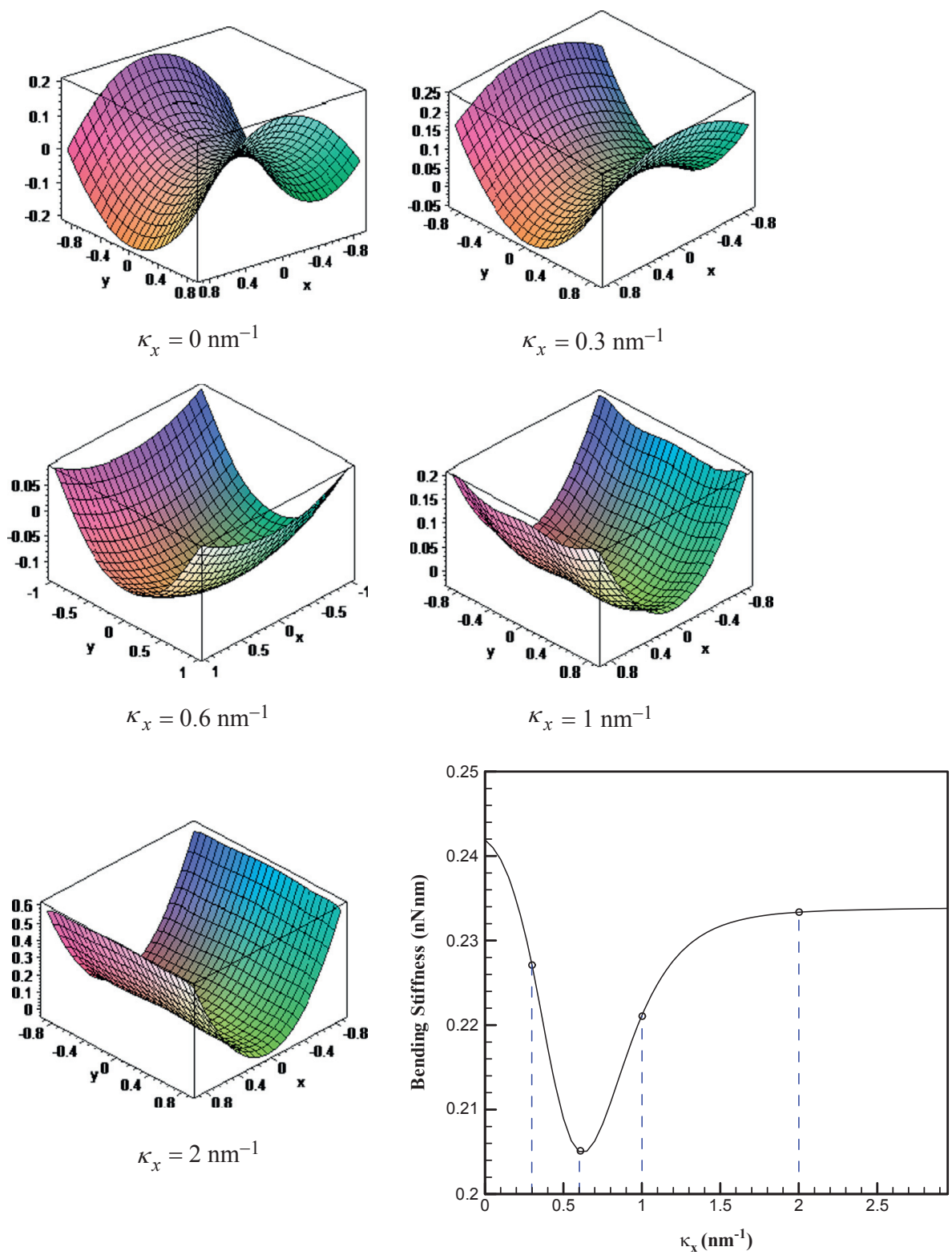

Fig. 5. The deformed shapes of monolayer graphene for different longitudinal curvatures $R_{x 0}=-2 \mathrm{~nm}, R_{y 0}=2 \mathrm{~nm}$. 


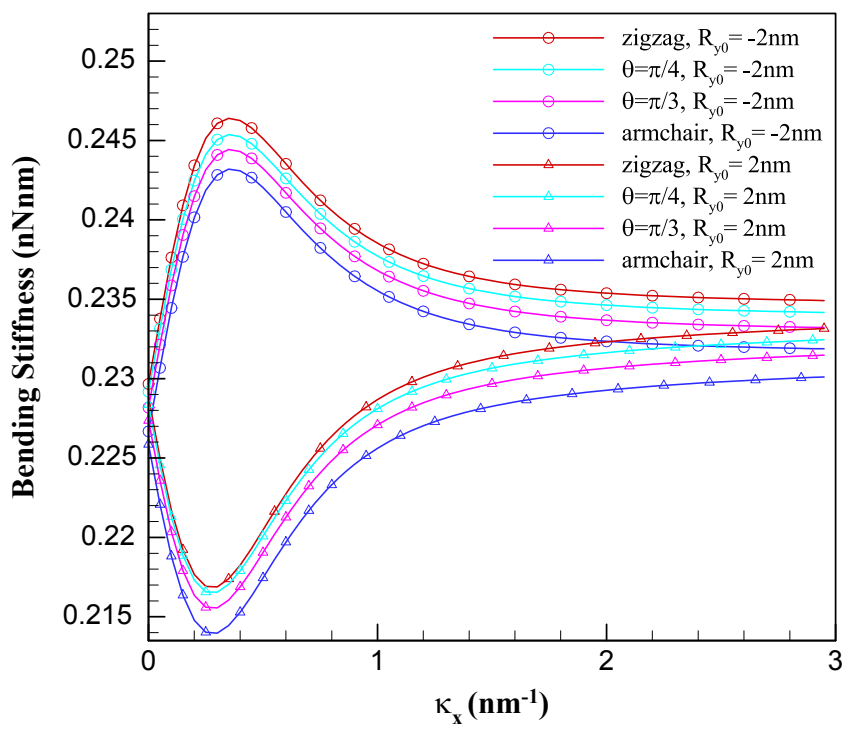

Fig. 6. Variation of the bending stiffness of the monolayer graphene for different material chiralities.

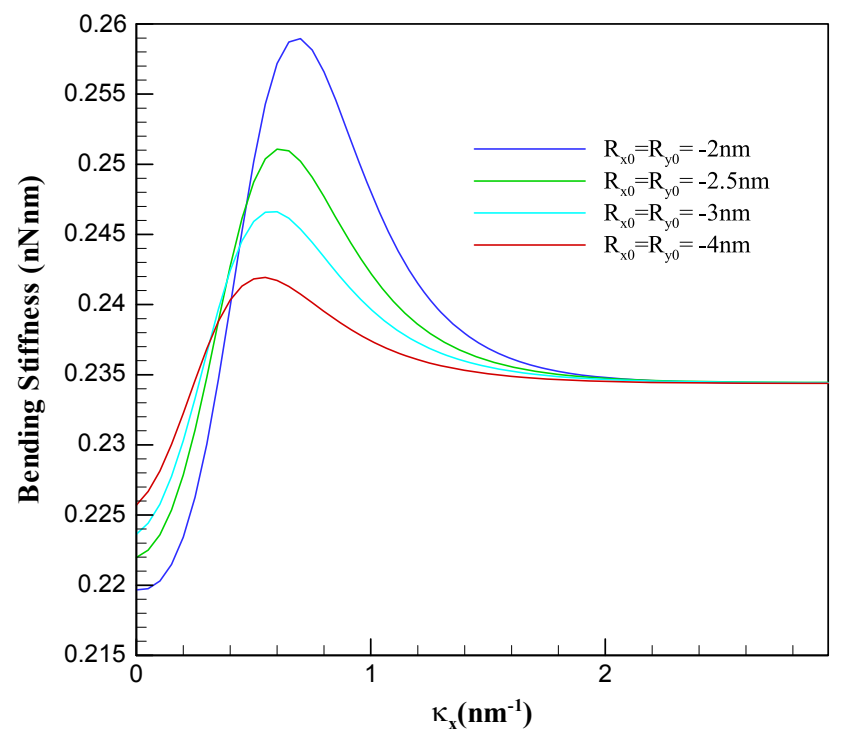

Fig. 7. Variation of the bending stiffness of a zigzag graphene sheet versus longitudinal curvature, for different initial curvatures.

The bending stiffness versus the longitudinal curvature is depicted in Fig. 7 for several initial curvatures. It can be seen that the maximum bending stiffness occurs at different values of curvature and it increases by decreasing the initial radii of curvatures. However, we found that a monolayer graphene with high initial curvatures in the same directions has the smallest stiffness at low values of longitudinal curvatures.

Variation of the bending moment versus the small scale effect is depicted in Fig. 8 for an initially flat monolayer graphene. It can be seen that in this special case the bending moment ratio increases by increasing the nonlocal parameter.

\section{Conclusion}

A study on the bending stiffness of the monolayer graphene with an initial curvature has been presented. The small scale effect has been considered using nonlocal elasticity. The deflection of the

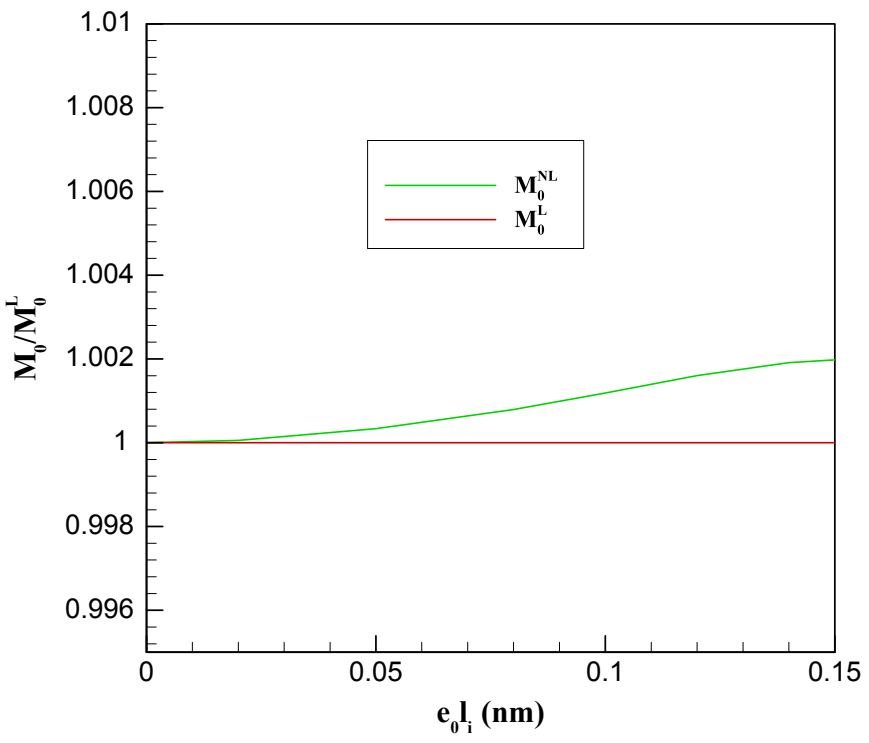

Fig. 8. Effect of nonlocal parameter on the bending moment of armchair graphene $\left(R_{x 0}=R_{y 0}=\right.$ infinite, $\left.R_{x}=1 \mathrm{~nm}\right)$.

graphene has been assumed to be large and an orthotropic constitutive law has been used for the monolayer graphene. The nonlinear equilibrium governing equations have been obtained and solved analytically and an explicit formulation has been defined for the bending stiffness of graphene. The effects of chiral angle, initial curvature and large deflection on the bending stiffness of graphene sheet have been shown. The bending stiffness of the graphene strongly depends on the initial curvature suggesting a more complex behavior than previously reported in the literature. Local maxima and minima are obtained in the bending stiffness versus curvature, suggesting the possibility of a smart tuning.

\section{Acknowledgment}

The financial support of Iran Nanotechnology Initiative Council is gratefully acknowledged. The research related to these results has also received funding from European Research Council under the European Union's Seventh Framework Programme (FP7/20072013)/ERC Grant agreement no. [279985] (ERC StG BIHSNAM, to NMP).

\section{References}

[1] A.K. Geim, K.S. Novoselov, The rise of graphene, Nature Materials 6 (2007) 183-191.

[2] X.L. Gao, K. Li, A shear-lag model for carbon nanotube-reinforced polymer composites, International Journal of Solids and Structures 42 (2005) 1649-1667.

[3] J.C. Meyer, A.K. Geim, M.I. Katsnelson, K.S. Novoselov, T.J. Booth, S. Roth, The structure of suspended graphene sheets, Nature 446 (2007) 60-63.

[4] C. Lee, X. Wei, J.W. Kysar, J. Hone, Measurement of the elastic properties and intrinsic strength of monolayer graphene, Science 321 (2008) 385-388.

[5] P.H. Tan, W.P. Han, W.J. Zhao, Z.H. Wu, K. Chang, H. Wang, Y.F. Wang, N. Bonini, N. Marzari, N. Pugno, G. Savini, A. Lombardo, A.C. Ferrari, The shear mode of multilayer graphene, Nature Materials 11 (2012) 294-300.

[6] K.N. Kudin, G.E. Scuseria, C2F, BN and C nanoshell elasticity from ab initio computations, Physical Review B 64 (2001) 235406.

[7] X. Shi, B. Peng, N.M. Pugno, H. Gao, Stretch-induced softening of bending rigidity in graphene, Applied Physics Letters 100 (2012) 191913.

[8] S.S. Terdalkar, S. Zhang, J.J. Rencis, K.J. Hsia, Molecular dynamics simulations of ion-irradiation induced deflection of 2D graphene films, International Journal of Solids and Structures 45 (2008) 3908-3917.

[9] X. Shi, N.M. Pugno, Y. Cheng, H. Gao, Gigahertz breathing oscillators based on carbon nanoscrolls, Applied Physics Letters 95 (2009) 163113. 
[10] N. Kacem, S. Baguet, S. Hentz, R. Dufour, Computational and quasi-analytical models for non-linear vibrations of resonant MEMS and NEMS sensors, International Journal of Non-Linear Mechanics 46 (2011) 532-542.

[11] Q. Lu, R. Huang, Nonlinear mechanics of single-atomic-layer graphene sheets, International Journal of Applied Mechanics 1 (2009) 443-467.

[12] Y. Huang, J. Wu, K.C. Hwang, Thickness of graphene and single-wall carbon nanotubes, Physical Review B 74 (2006) 245413.

[13] N.M. Pugno, Young's modulus reduction of defective nanotubes, Applied Physics Letters 90 (2007) 043106.

[14] Q. Lu, M. Arroyo, R. Huang, Elastic bending modulus of monolayer graphene, Journal of Physics D: Applied Physics 42 (2009) 102002.

[15] J.W. Jiang, J.S. Wang, B. Li, Elastic and nonlinear stiffness of graphene: a simple approach, Physical Review B 81 (2010) 073405.

[16] F. Scarpa, S. Adhikari, A.J. Gil, C. Remillat, The bending of single layer graphene sheets: the lattice versus continuum approach, Nanotechnology 21 (2010) 125702.

[17] M. Arroyo, T. Belytschko, An atomistic-based finite deformation membrane for single layer crystalline films, Journal of the Mechanics and Physics of Solids 50 (2002) 1941-1977.

[18] M. Arroyo, T. Belytschko, A finite deformation membrane based on interatomic potentials for the transverse mechanics of nanotubes, Mechanics of Materials 35 (2003) 193-215.

[19] E. Cadelano, P.L. Palla, S. Giordano, L. Colombo, Nonlinear elasticity of monolayer graphene, Physical Review Letters 102 (2009) 235502.

[20] Q. Lu, R. Huang, Nonlinear mechanics of single-atomic-layer graphene sheets, International Journal of Applied Mechanics 1 (2009) 443467.

[21] C.T. Sun, H. Zhang, Size-dependent elastic moduli of platelike nanomaterials, Journal of Applied Physics 93 (2003) 1212.
[22] Q. Wang, Effective in-plane stiffness and bending rigidity of armchair and zigzag carbon nanotubes, International Journal of Solids and Structures 41 (2004) 5451-5461.

[23] A.C. Eringen, On differential equations of nonlocal elasticity and solutions of screw dislocation and surface waves, Journal Applied Physics 54 (1983) 4703-4710.

[24] A.C. Eringen, Nonlocal Continuum Field Theories, Springer, New York, 2002.

[25] Y. Chen, J.D. Lee, A. Eskandarian, Atomistic viewpoint of the applicability of microcontinuum theories, International Journal of Solids and Structures 41 (2004) 2085-2097.

[26] E. Jomehzadeh, A.R. Saidi, Decoupling the nonlocal elasticity equations for three dimensional vibration analysis of nano-plates, Composite Structures 93 (2011) 1015-1020.

[27] E. Jomehzadeh, A.R. Saidi, A study on large amplitude vibration of multilayered graphene sheets, Computational Materials Science 50 (2011) 1043-1051.

[28] E. Jomehzadeh, A.R. Saidi, N.M. Pugno, Large amplitude vibration of a bilayer graphene embedded in a nonlinear polymer matrix, Physica E: Lowdimensional Systems and Nanostructures 44 (2012) 1973-1982.

[29] O.A. Shenderova, V.V. Zhirnov, D.W. Brenner, Carbon nanostructures, Critical Reviews in Solid State and Materials Sciences 27 (2002) 227-356.

[30] Z.P. Bazant, L. Cedolin, Stability of Structures Elastic, Inelastic, Fracture, and Damage Theories, Dover, New York, 2003.

[31] V. Sundararaghavan, A. Waas, Non-local continuum modeling of carbon nanotubes: physical interpretation of non-local kernels using atomistic simulations, Journal of the Mechanics and Physics of Solids 59 (2011) 1191-1203. 